\title{
Dermatofibroma celular. Reporte de un caso
}

\section{Cellular dermatofibroma. A case report}

Virginia García ${ }^{1 *}$, María P. Gaggino ${ }^{1}$ Iliana S. Garay ${ }^{1}$, María V. Laborié2 y Alejandro Ruiz-Lascano

${ }^{1}$ Servicio de Dermatología; ${ }^{2}$ Servicio de Patología. Hospital Privado Universitario de Córdoba, Universidad Católica de Córdoba, Córdoba, Argentina

\section{Resumen}

Los dermatofibromas son tumores histiofibrosos benignos de origen dérmico. Desde el punto de vista clínico e histológico pueden clasificarse en múltiples variantes morfológicas, entre ellas el tipo celular, que es una variable poco frecuente. Los dermatofibromas celulares tienden a presentarse en pacientes adultos y se localizan principalmente en los miembros y con menor frecuencia en el tronco. Si bien se trata de una afección benigna, el tratamiento de elección es la escisión completa debido a su probabilidad de recurrir localmente y rara vez metastatizar.

Palabras clave: Dermatofibroma. Histiocitoma fibroso celular. Tumor dérmico.

\section{Abstract}

Dermatofibromas are benign histiofibrous tumors of dermal origin. According to the clinical and histological point of view, it can be classified into multiple variables, including the cell type that is treated with a rare variable. Cellular dermatofibromas offer to occur in adult patients and are located mainly in the limbs and less frequently in the trunk. Although it is a benign entity, the treatment of choice is complete excision of it due to its likelihood of recurring locally and rarely metastasizing.

Keywords: Dermatofibroma. Cellular fibrous histiocytoma. Dermal tumor.

\section{Introducción}

Los dermatofibromas celulares son un subtipo de dermatofibromas caracterizados desde un punto de vista histológico por su hipercelularidad. Se trata de tumoraciones cutáneas benignas, originadas en la der$\mathrm{mis}^{1,2}$. Suelen presentarse en pacientes adultos, con un ligero predominio en mujeres, y se localizan principalmente en los miembros ${ }^{1,2}$.

A continuación, comunicamos el caso de un paciente de 72 años a quien, a partir de una lesión nodular ulcerada en el hombro, se le diagnosticó dermatofibroma celular, por lo que se realizó su extirpación completa para evitar recaídas, necrosis y metástasis.

\section{Caso clínico}

Se presentó a la consulta con el servicio de dermatología un varón de 72 años, sin antecedentes patológicos de relevancia. El motivo de consulta era una tumoración asintomática en el hombro derecho, de crecimiento progresivo, de 8 meses de evolución.

En la exploración física se observó una lesión nódulo-tumoral eritemato-violácea con una ulceración
Correspondencia:

*Virginia García

E-mail: virginiagarciatosello@gmail.com

DOI: 10.24875/MCUT.21000037 licencia CC BY-NC-ND (http://creativecommons.org/licenses/by-nc-nd/4.0/). 


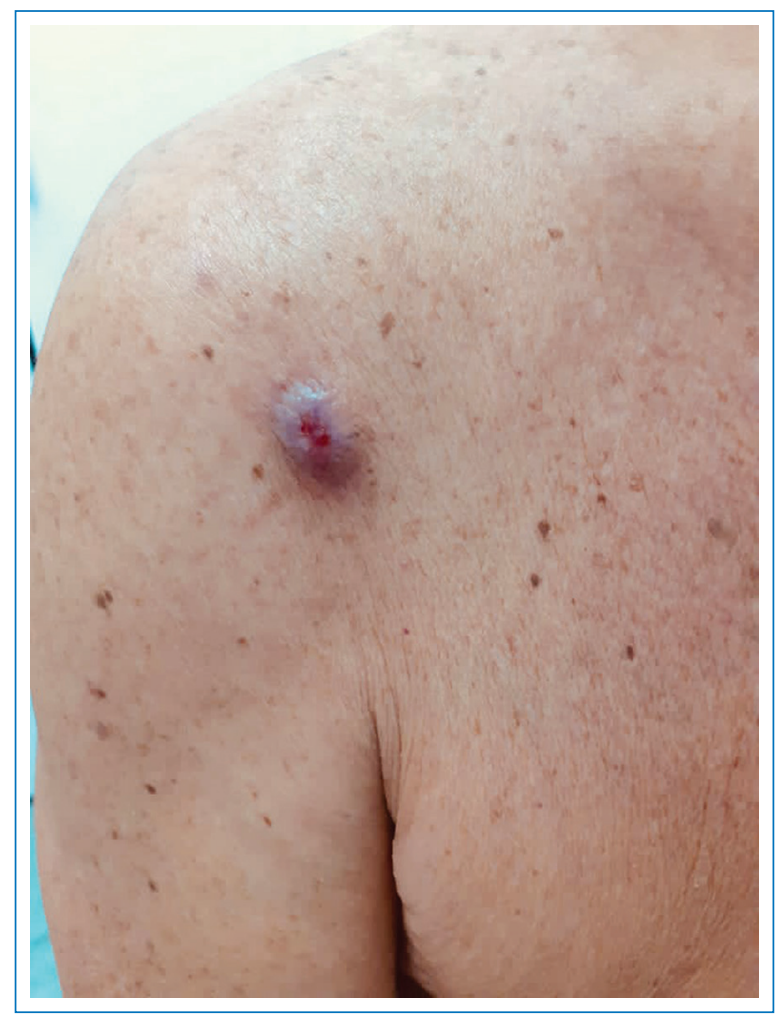

Figura 1. Imagen clínica. Tumoración eritemato-violácea con ulceración central en el hombro derecho.

central de $3 \mathrm{~cm}$ de diámetro, de consistencia indurada, firme y no dolorosa a la palpación (Fig. 1). Dermatoscópicamente se objetivaba solución de continuidad en el centro, con áreas de fibrosis y vasos atípicos (Fig. 2). En el resto de la exploración física el paciente no presentaba otras lesiones de relevancia ni adenopatías palpables.

Planteando como diagnósticos diferenciales carcinoma basocelular ulcerado, dermatofibrosarcoma protuberans y leiomiosarcoma, se realizó extirpación en losange con márgenes de $2 \mathrm{~mm}$ y estudio histológico de la pieza (P:7500/20). El estudio reveló epidermis hiperplásica con superficie escamocostrosa fibrinoleucocitaria y necrosis central, dermis con células fusadas y fibras de colágeno engrosadas e intensamente eosinófilas atrapadas, y márgenes profundos comprometidos (Fig. 3).

Se solicitaron marcadores SOX 10 y $\mathrm{p} 40$, que fueron ambos negativos. La inmunomarcación fue positiva para CD68 en toda la lesión y de manera focal para ASMA (Figs. 4 a 6), y presentaba un índice de proliferación (Ki 67) del 3\%, descartando malignidad de la pieza y arribando al diagnóstico de dermatofibroma

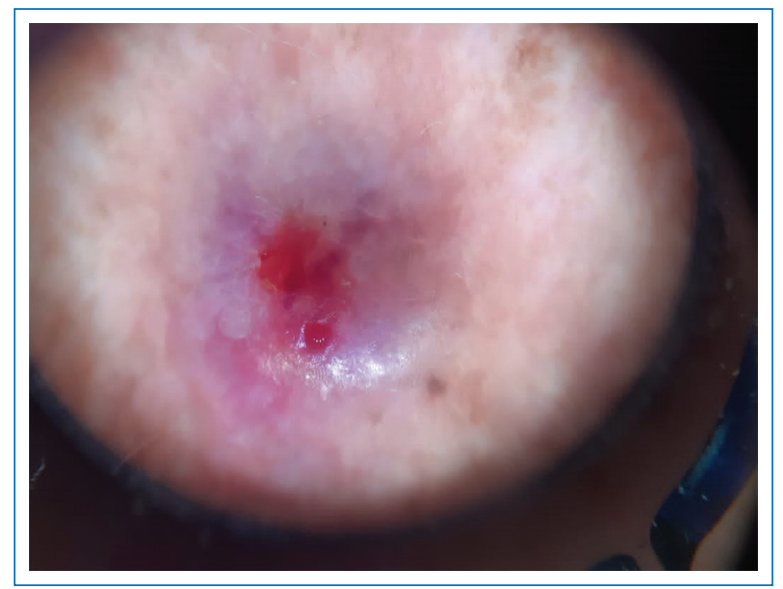

Figura 2. Imagen dermatoscópica. Obsérvese la fibrosis periférica con solución de continuidad central.

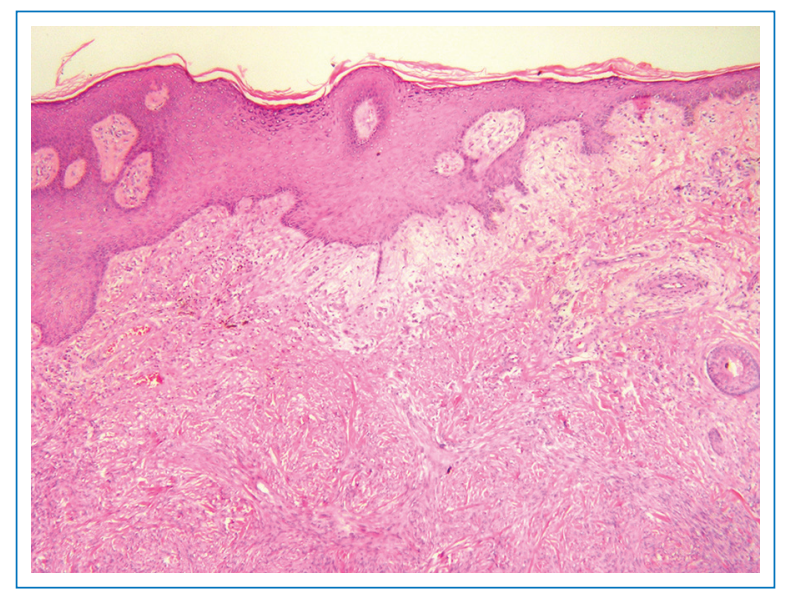

Figura 3. Imagen microscópica (H-E 4×). Nótese en la dermis la presencia de células fusadas y haces eósinofilos de colágeno engrosado.

celular focalmente ulcerado, en correlación con la clínica.

Debido al compromiso de uno de los márgenes y a la alta tasa de recurrencia que pueden presentar estas lesiones, se realizó una ampliación de los márgenes de la lesión.

El paciente evolucionó favorablemente y en la actualidad se encuentra en seguimiento sin haber presentado nuevas lesiones de naturaleza similar.

\section{Discusión}

Los dermatofibromas son tumores fibrohistiocitarios indolentes muy comunes en la práctica dermatológica. Se trata de una afección benigna y recurrente. Se han 


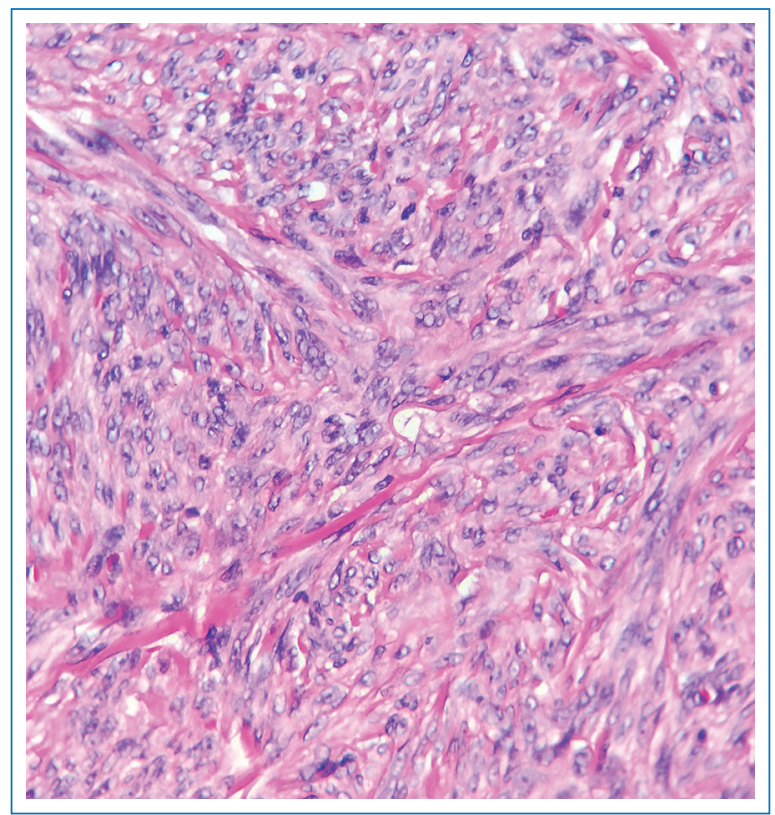

Figura 4. Imagen microscópica (H-E 40×). A mayor aumento se observan las características células fusadas y los haces eosinófilos.

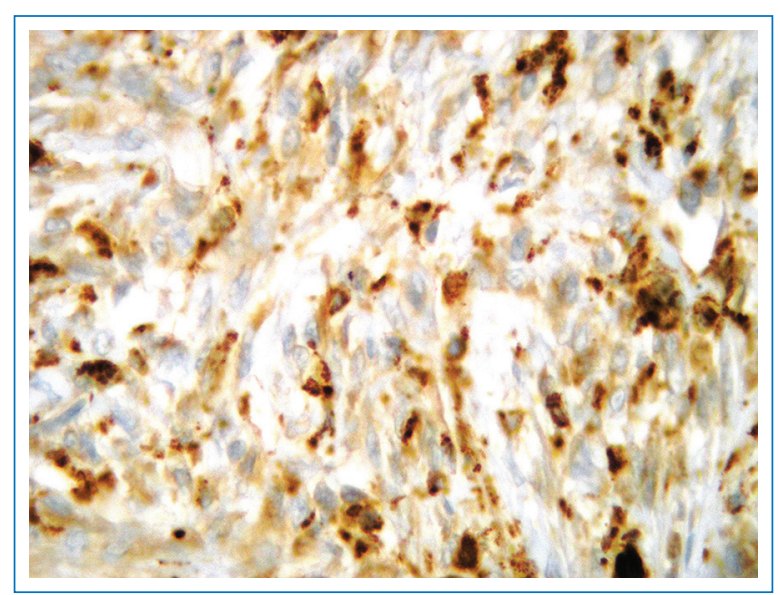

Figura 5. Marcación para CD 68 con resultado positivo.

identificado múltiples variantes de acuerdo con las características histológicas: aneurismática, hemosiderótica, epitelioide, atípica, lipídica, de células claras, en empalizada, atrófico, queloideo, de células granulares, mixoide, liquenoide, de células balonizadas y la variante celular o histiocitoma fibroso, que representa aproximadamente el $5 \%$ de todos los dermatofibromas ${ }^{1-3}$. Al igual que en los dermatofibromas clásicos, la variante celular es más frecuente en mujeres adultas, con un promedio de edad de 42 años ${ }^{1,2}$.

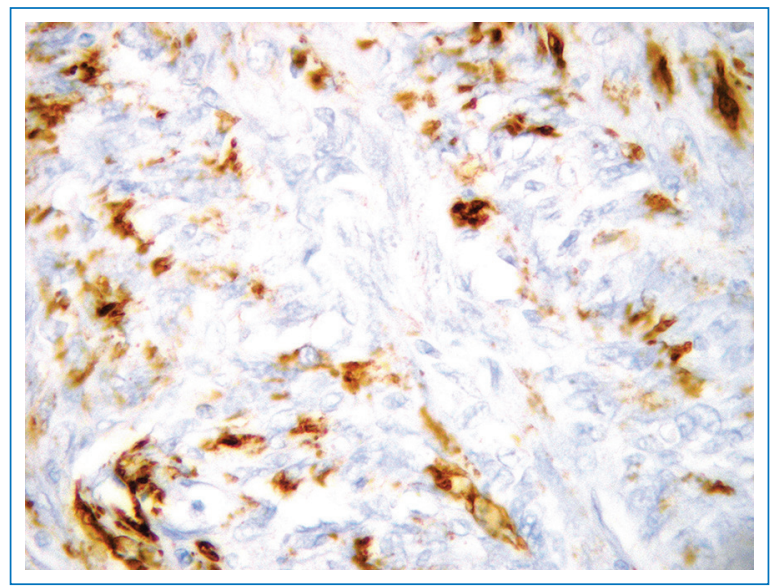

Figura 6. ASMA positivo focal.

Clínicamente se trata de un nódulo, placa o pápula dérmico único, firme y de crecimiento lento, cuyo color varía entre rojo-amarronado y violáceo. Puede medir entre 2 y $50 \mathrm{~mm}^{1}$. La localización más frecuente es en los miembros superiores $(41 \%)^{1}$, aunque también pueden aparecer en los miembros inferiores, la cabeza, el cuello y el tronco $0^{1,2,4}$.

A la dermatoscopía se puede observar tejido fibroso generalmente central y pigmento periférico amarronado ${ }^{3-5}$, y en otros casos pigmentación azul grisácea sobre toda la lesión ${ }^{6}$, similar a lo observado en nuestro paciente, que también presentaba ulceración central.

Los principales diagnósticos diferenciales son dermatofibrosarcoma protuberans, leiomiosarcoma, melanoma, quistes y carcinomas (principalmente basocelular nodular), de los que se diferencia principalmente a partir de la histología y la inmunomarcación ${ }^{1,6}$ (Tabla 1).

Histológicamente, los dermatofibromas celulares se caracterizan por una densa proliferación de células fibrohistiocíticas de citoplasma eosinófilo y núcleos ahusados que se disponen en un patrón estoriforme entre los haces de colágeno. Se observa acantosis y en ocasiones hiperqueratosis y pigmentación de la basal. Es frecuente hallar un notable componente vascular y en algunos casos invasión de tejido subcutáneo y aumento del número de mitosis. El $10-12 \%$ pueden mostrar necrosis central o ulceración ${ }^{2,6,7}$.

La técnica de inmunohistoquímica suele mostrar positividad variable para CD68 y ASMA, siendo útil solicitar otros marcadores (CD34, desmina, S100 y factor XIIla) para diferenciarlo de otras afecciones clínicamente similares ${ }^{1,2,6,7}$. 
Tabla 1. Diagnósticos diferenciales

\begin{tabular}{|c|c|c|c|c|}
\hline Tumor & Clínica & Dermatoscopía & Histología & Inmunohistoquímica \\
\hline $\begin{array}{l}\text { Dermatofibroma } \\
\text { celular }\end{array}$ & $\begin{array}{l}\text { Nódulo firme, } \\
\text { hiperpigmentado, } \\
\text { eritemato-violáceo } \\
0,2-5 \mathrm{~cm}\end{array}$ & $\begin{array}{l}\text { Fibrosis central } \\
\text { Pseudored periférica }\end{array}$ & $\begin{array}{l}\text { Hipercelularidad } \\
\text { fibrohistiocítica, } \\
\text { citoplasma eosinófilo y } \\
\text { núcleos ahusados } \\
\text { Abundante vascularización } \\
\text { Ocasional necrosis }\end{array}$ & $\begin{array}{l}\text { CD68+, ASMA+, } \\
\text { desmina+, XIIIa+ }\end{array}$ \\
\hline $\begin{array}{l}\text { Dermatofibrosarcoma } \\
\text { protuberans }\end{array}$ & $\begin{array}{l}\text { Nódulo/parche firme, } \\
\text { violáceo, eritematoso o } \\
\text { amarronado } \\
1-5 \mathrm{~cm}\end{array}$ & $\begin{array}{l}\text { Red pigmentada } \\
\text { periférica con fondo } \\
\text { rosado/eritematoso } \\
\text { Abundante } \\
\text { vascularización }\end{array}$ & $\begin{array}{l}\text { Hipercelularidad } \\
\text { Células dendríticas con } \\
\text { contenido melánico } \\
\text { Núcleos hipercromáticos y } \\
\text { escaso citoplasma } \\
\text { eosinófilo }\end{array}$ & CD34+ \\
\hline Leiomiosarcoma & $\begin{array}{l}\text { Pápulas, nódulos o placas } \\
\text { del color de la piel, } \\
\text { eritematosas o azules } \\
\text { Superficie irregular o } \\
\text { ulcerosa } \\
0,3-3 \mathrm{~cm}\end{array}$ & $\begin{array}{l}\text { Inespecífica } \\
\text { Abundantes vasos } \\
\text { atípicos }\end{array}$ & $\begin{array}{l}\text { Células fusiformes atípicas } \\
\text { con citoplasma } \\
\text { homogéneo eosinófilo } \\
\text { Núcleos en forma de } \\
\text { "cigarro" } \\
\text { Células tumorales } \\
\text { dispuestas en fascículos o } \\
\text { nódulos }\end{array}$ & ASMA + , desmina+ \\
\hline $\begin{array}{l}\text { Carcinoma } \\
\text { basocelular nodular }\end{array}$ & $\begin{array}{l}\text { Pápula-nódulo eritematoso } \\
\text { de tamaño variable }\end{array}$ & $\begin{array}{l}\text { Vasos arboriformes } \\
\text { Puntos azul-grisáceos } \\
\text { de distribución irregular }\end{array}$ & $\begin{array}{l}\text { Infiltración en forma de } \\
\text { nido con células } \\
\text { basaloides }\end{array}$ & $\begin{array}{l}\mathrm{Bcl}-2+, \mathrm{CD} 10+ \\
\mathrm{CEA}+\end{array}$ \\
\hline Melanoma nodular & $\begin{array}{l}\text { Nódulo negruzco } \\
6 \mathrm{~mm} \mathrm{a}>1 \mathrm{~cm}\end{array}$ & $\begin{array}{l}\text { Glóbulos } \\
\text { azul-negruzcos } \\
\text { Red pigmentada o } \\
\text { pseudored } \\
\text { Vasos en forma de } \\
\text { coma }\end{array}$ & $\begin{array}{l}\text { Melanocitos atípicos con } \\
\text { expansión vertical } \\
\text { Núcleos hipercromáticos, } \\
\text { grandes y nucleolos } \\
\text { prominentes } \\
\text { Compromiso dérmico con } \\
\text { o sin compromiso } \\
\text { epidérmico }\end{array}$ & $\mathrm{S} 100_{+}$ \\
\hline
\end{tabular}

El tratamiento sugerido es la extirpación completa de la lesión, ya sea mediante losange o cirugía de Mohs, aunque al tratarse de una afección benigna puede hacerse un seguimiento estrecho'.

La tasa de recurrencia local de estos tumores es elevada (13-26\%), sobre todo cuando el tamaño de la lesión es $>1 \mathrm{~cm}^{1,2,7,8}$. Ocurren principalmente sobre lesiones biopsiadas o resecadas de forma incompleta. Esto fue lo que nos motivó a realizar una ampliación en nuestro paciente para asegurar unos márgenes libres ${ }^{1,2}$.

En raras ocasiones se han reportado metástasis en los ganglios linfáticos o el pulmón ${ }^{2,8-10}$. El riesgo de metástasis depende fundamentalmente del tamaño del tumor primario, de la hipercelularidad y de las recurrencias locales; sin embargo, teniendo en cuenta su benignidad, las metástasis se comportan de forma indolente ${ }^{9}$.
El interés de este reporte radica en presentar una variable poco común de una afección benigna frecuente.

\section{Financiamiento}

No se contó con fuentes externas de financiamiento.

\section{Conflicto de intereses}

Los autores declaran no tener ningún conflicto de intereses.

\section{Responsabilidades éticas}

Protección de personas y animales. Los autores declaran que para esta investigación no se han realizado experimentos en seres humanos ni en animales. 
Confidencialidad de los datos. Los autores declaran que han seguido los protocolos de su centro de trabajo sobre la publicación de datos de pacientes.

Derecho a la privacidad y consentimiento informado. Los autores han obtenido el consentimiento informado de los pacientes y/o sujetos referidos en el artículo. Este documento obra en poder del autor de correspondencia.

\section{Bibliografía}

1. Gaufin M, Michaelis T, Duffy K. Cellular dermatofibroma: clinicopathologic review of 218 cases of cellular dermatofibroma to determine the clinical recurrence rate. Dermatol Surg. 2019;45:1359-64.

2. Alves JV, Matos DM, Barreiros HF, Bártolo EA. Variants of dermatofibroma - a histopathological study. An Bras Dermatol. 2014;89: 472-7.
3. Kelati A, Aqil N, Baybay H, Gallouj S, Mernissi FZ. Beyond classic dermoscopic patterns of dermatofibromas: a prospective research study. J Med Case Rep. 2017;11:266.

4. Kilinc Karaarslan I, Gencoglan G, Akalin T, Ozdemir F. Different dermoscopic faces of dermatofibromas. J Am Acad Dermatol. 2007;57:401-6.

5. Zaballos P, Puig S, Llambrich A, Malvehy J. Dermoscopy of dermatofibromas: a prospective morphological study of 412 cases. Arch Dermatol. 2008;144:75-83.

6. 囚enel E, Yuyucu Karabulut Y, Do囚ruer 囚enel S. Clinical, histopathological, dermatoscopic and digital microscopic features of dermatofibroma: a retrospective analysis of 200 lesions. J Eur Acad Dermatol Venereol. 2015;29:1958-66.

7. Calonje E, Mentzel T, Fletcher CD. Cellular benign fibrous histiocytoma. Clinicopathologic analysis of 74 cases of a distinctive variant of cutaneous fibrous histiocytoma with frequent recurrence. Am J Surg Pathol. 1994;18:668-76.

8. Kaddu S, McMenamin ME, Fletcher CD. Atypical fibrous histiocytoma of the skin: clinicopathologic analysis of 59 cases with evidence of infrequent metastasis. Am J Surg Pathol. 2002;26:35-46.

9. Colome-Grimmer MI, Evans HL. Metastasizing cellular dermatofibroma A report of two cases. Am J Surg Pathol. 1996;20:1361-7.

10. Guillou L, Gebhard S, Salmeron M, Coindre JM. Metastasizing fibrous histiocytoma of the skin: a clinicopathologic and immunohistochemical analysis of three cases. Mod Pathol. 2000;13:654-60. 